\title{
Coste-efectividad del tratamiento de síntomas vasomotores en cáncer de mama con desvenlafaxina en México
}

\author{
Miguel Angel Ramírez • Gustavo Peniche • \\ Carlos Nuño · Joaquín Federico Mould-Quevedo
}

Published online: 16 April 2013

(C) The Author(s) 2013. This article is published with open access at Springerlink.com

Resumen Objetivo Estimar el coste-efectividad del manejo de síntomas vasomotores (SVM) con terapia de reemplazo hormonal (TRH) y alternativas no hormonales en pacientes de cáncer de mama (CaMa), desde la perspectiva institucional mexicana.

Métodos Se realizó un modelo Markov para estimar consecuencias de salud y económicas a 5 años (ciclos trimestrales). Las medidas de efectividad fueron: reducción en bochornos y años de vida ajustados por calidad ganados (AVAC's). Se compararon: medroxiprogesterona + estrógenos conjugados $(2,5 \mathrm{mg} / 0,625 \mathrm{mg} / \mathrm{día})$, tibolona ( $2,5 \mathrm{mg}$ /día), estrógenos conjugados (EC, 0,625 mg/día) y ningún tratamiento. Desvenlafaxina (100 mg/día) fue la referencia no hormonal. La efectividad de las terapias se extrajo de documentos publicados. El perfil de uso de recursos se obtuvo de revisión retrospectiva de expedientes $(n=140)$ de pacientes del Instituto Mexicano del Seguro Social. Los costes se extrajeron de fuentes oficiales. Se construyeron curvas de aceptabilidad.

Resultados En las pacientes sin contraindicación de TRH, desvenlafaxina tuvo el menor coste (entre US\$3.011,46 y US\$3.169,29) y los mayores niveles de efectividad y utilidad (al menos 441 bochornos menos y 0,16 AVAC's más que con EC). En pacientes con TRH contraindicada, desvenlafaxina es más costosa que el no tratamiento (US\$1.186), pero evitó 2.754 bochornos y ganó 0,75 AVAC's. Las curvas de

M.A. Ramírez · G. Peniche · C. Nuño

CPP Customized Premium Products S.A. de C.V.,

San Bernandino No. 17 (Insurgentes Sur), Mexico City,

Distrito Federal 03810, Mexico

J.F. Mould-Quevedo ( $\square)$

Latin America and Primary Care Emerging Market Business Unit,

Pfizer Inc., 235 East 42nd Street, New York, NY 10017, USA

e-mail: joaquin.mould@pfizer.com aceptabilidad muestran que desvenlafaxina es la terapia no hormonal con la mayor probabilidad de ser coste-efectiva.

Conclusiones Desvenlafaxina constituye una alternativa no hormonal coste-ahorradora y coste-efectiva para el manejo de SVM en pacientes con CaMa sin y con contraindicación de uso de TRH, respectivamente.

Palabras clave Cáncer de mama Bochornos .

Desvenlafaxina $\cdot$ México $\cdot$ Evaluación económica

\begin{abstract}
Objective Estimate the cost-effectiveness of vasomotor symptoms (VMS) management with hormone replacement therapy (HRT) and non-hormonal alternatives in patients with breast cancer (BC), regarding the Mexican institutional perspective.

Methods A Markov model was developed to estimate health and economic consequences until five years (quarterly cycles). Effectiveness measures were: reduction in hot flashes and quality-adjusted life years gained (QALY's). Alternatives compared were: conjugated estrogens + medroxyprogesterone $(2.5 \mathrm{mg} / 0.625 \mathrm{mg} /$ day $)$, tibolone ( $2.5 \mathrm{mg} /$ day $)$, conjugated estrogens (CE, $0.625 \mathrm{mg} /$ day) and no treatment. Desvenlafaxine (100 mg/day) was the non-hormonal reference. The effectiveness of the therapies was extracted from published literature. The resource use profile was obtained from retrospective review of clinical files $(n=140)$ of patients attending Instituto Mexicano del Seguro Social. Costs were extracted from official sources. Acceptability curves were constructed.

Results In patients with no contraindication to HRT, desvenlafaxine had the lowest cost (between US\$3.011,46 and US $\$ 3.169,29)$ and higher levels of effectiveness and utility (at least 441 fewer hot flashes and 0.16 QALY's more than $\mathrm{CE}$ ). In patients with HRT contraindicated, desvenlafaxine
\end{abstract}


is more expensive than no treatment (US\$1.186), but prevented 2.754 hot flashes and gained 0.75 QALY's. Acceptability curves show that desvenlafaxine is the non-hormonal therapy most likely to be cost effective.

Conclusions Desvenlafaxine is a cost-saving and cost effective non-hormonal alternative in the management of VMS in both, BC patients without and with contraindication to HRT use, respectively.

Keywords Breast cancer · Hot flashes - Desvenlafaxine . Economic evaluation $\cdot$ Mexico

\section{Introducción}

El término "síntomas vasomotores" (SVM) se utiliza para referirse a varios síntomas asociados a la menopausia y que abarca tanto a los bochornos como a la sudoración nocturna [1]. Se ha estimado que $19 \%, 36,9 \%, 56,8 \%$ y $48,8 \%$ de las mujeres que se encuentran en la etapa premenopáusica, perimenopáusica temprana, perimenopáusica tardía y en la postmenopausia, respectivamente, presentan SVM [2]. En México, se realizó un estudio con mujeres sanas de entre 45 y 59 años en el que se observó que el 71,9 \% y el 22,6 \% de las mujeres en la etapa de postmenopausia experimentó bochornos y sudoración nocturna, respectivamente [3].

Las mujeres con cáncer de mama (CaMa) tratadas con quimioterapia adyuvante, terapia hormonal o una combinación de ambas a menudo sufren de una deficiencia de estrógenos, por lo que los síntomas climatéricos (entre los que se encuentran los SVM) pueden presentarse en mujeres premenopáusicas sometidas a este tratamiento y son de mayor intensidad en mujeres postmenopáusicas [4]. Mortimer y cols. [5] observaron que el $78 \%$ de mujeres con diagnóstico de cáncer de mama en etapas tempranas y que fueron tratadas con tamoxifeno (el cual inhibe la proliferación de células cancerosas promovida por el estrógeno), presentaron bochornos.

Para el manejo de SVM en mujeres sin antecedentes de carcinomas estrógeno dependientes (como el CaMa), las guías de práctica clínica recomiendan el uso de terapia de reemplazo hormonal (TRH) basada en el uso de estrógenos conjugados (EC), ya sea solos o en combinación con progesterona o un progestágeno $[1,6]$.

Sin embargo, el manejo farmacológico de SVM en mujeres sobrevivientes a CaMa o con diagnóstico de CaMa permanece a discusión: de acuerdo a Xydakis y cols. [4]: "Aunque la preocupación de que el riesgo puede ser superior al beneficio no debe ser definitivamente descartada, creemos que la mayoría de los datos retrospectivos y prospectivos permiten el uso de TRH en sobrevivientes de CaMa con síntomas climatéricos graves que tuvieron la enfermedad en estadio I o II, ganglios y receptor de estrógeno negativos y preferentemente con un intervalo libre de enfermedad mínimo de 2 años" [4].
Se ha estudiado el uso de otras alternativas farmacológicas no hormonales para el manejo de SVM. Entre ellas se encuentran los inhibidores selectivos de la recaptura de serotonina y norepinefrina, como la desvenlafaxina (DVS). Esta alternativa no hormonal presenta la ventaja de que puede usarse en el manejo de SVM en pacientes de CaMa dado que no se metaboliza por la vía de la isoenzima CYP2D6 del citocromo $\mathrm{P} 450$ y por lo tanto no limita el efecto del tamoxifeno [7].

Por otro lado, un aspecto que no se puede dejar de lado al considerar la selección de una terapia entre otras alternativas farmacológicas para el manejo de una enfermedad, es el de los costes asociados a cada una de ellas. Cuando, además del coste de adquisición de las terapias farmacológicas, se consideran los costes del manejo de los efectos indeseables de las mismas, consultas, hospitalizaciones, pruebas diagnósticas y estudios de gabinete, entre otros, se tiene un panorama que se aproxima mucho más al coste total asociado a una terapia farmacológica dada.

Cuando se consideran de manera conjunta las consecuencias en salud y los costes de varias alternativas farmacológicas y se comparan con las consecuencias de una terapia previamente definida como referencia, se habla de una evaluación económica completa del tipo coste-efectividad. Este tipo de evaluaciones han cobrado cada vez mayor importancia dada la necesidad de que las instituciones prestadoras de servicios de salud asignen sus recursos de manera cada vez más eficiente.

Se han publicado estudios de coste-efectividad del manejo de SVM con TRH (estrógenos conjugados, EC, + medroxiprogesterona, MPA; noretisterona + etinilestradiol y tibolona, TIB) en mujeres que no presentan CaMa [8-10]. En general, en estos estudios se ha estimado que la TRH constituye una alternativa coste-efectiva para el manejo de SVM.

A la fecha de la elaboración de la presente investigación, no se han publicado estudios que evalúen el costeefectividad del uso de DVS para el manejo de SVM en pacientes de CaMa. Ante esta situación, se llevó a cabo un análisis cuyo objetivo fue estimar las consecuencias clínicas y económicas derivadas del uso de DVS, EC (como monoterapia y en combinación con MPA) y TIB en el manejo de SVM en mujeres con CaMa, desde la perspectiva de una institución representativa del sector salud en México, como es el Instituto Mexicano del Seguro Social (IMSS).

\section{Métodos}

Presentación de modelo

Se desarrolló un modelo de Markov para estimar el costeefectividad de diversas alternativas terapéuticas en el manejo de SVM en pacientes con antecedentes de CaMa o con 
Figura 1 Estados de salud considerados en el modelo de manejo de síntomas vasomotores (SVM) en pacientes con cáncer de mama (CaMa)

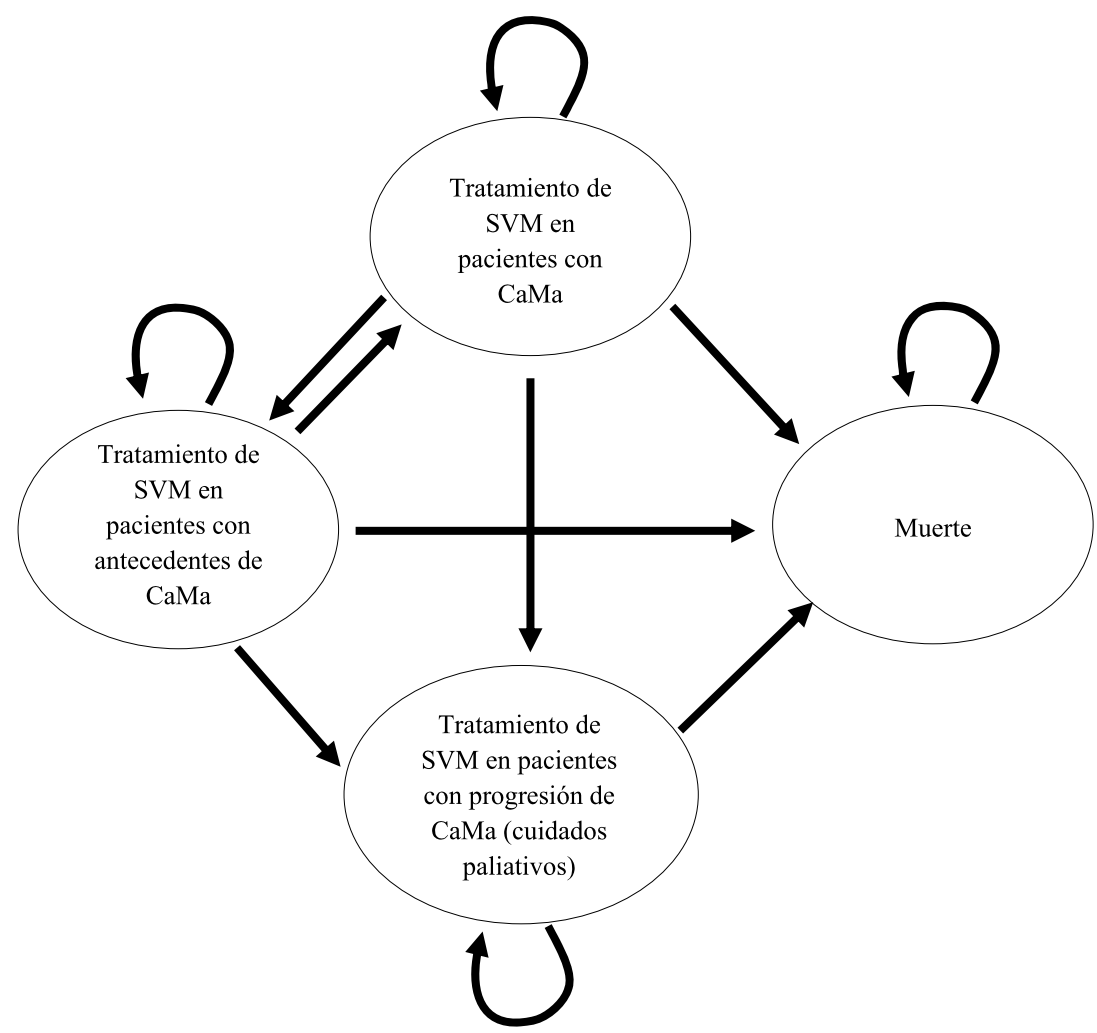

diagnóstico del mismo. Se consideraron distintos perfiles en la población que ingresa en el modelo de acuerdo al tipo de marcador hormonal y la presencia de útero. El estudio fue realizado considerando la perspectiva del IMSS al estimar el coste médico directo (el agregado del coste de adquisición de medicamentos, consultas médicas, hospitalización, pruebas de diagnóstico, estudios de gabinete y manejo de eventos adversos) en el que incurre esta institución al tratar los SVM en la población objetivo con las alternativas consideradas en el análisis durante un periodo de 5 años.

\section{Población objetivo}

La población objetivo del presente estudio se encuentra constituida por pacientes que están en tratamiento para Ca$\mathrm{Ma}$, pacientes de CaMa sometidos a cuidados paliativos y pacientes con antecedentes de CaMa. Estos tres grupos son susceptibles de ser sometidos o no a tratamiento farmacológico para manejo de SVM. El agente farmacológico empleado en el manejo de SVM se asigna en función de la presencia de receptores hormonales positivos: pacientes sin estos receptores son susceptibles de ser tratadas con TRH o alternativas no hormonales (DVS), mientras que pacientes con receptores hormonales positivos tienen contraindicado el uso de TRH y pueden ser sometidas solo a las alternativas no hormonales $[1,7]$.
Alternativas en comparación

Se compararon alternativas farmacológicas con indicación para el manejo de SVM que cuentan con clave en el Cuadro Básico y Catálogo de Medicamentos [11]: MPA/EC (2,5 mg/0,625 mg por día), TIB (2,5 mg por día) y EC (0,625 mg/día). Las dosis especificadas son las que se indican en las guías de práctica clínica y Cuadro Básico y Catálogo de Medicamentos [7, 11]. Adicionalmente se consideró como alternativa la opción de no aplicar esquema farmacológico alguno. La alternativa no hormonal de referencia fue DVS, a una dosis de $100 \mathrm{mg} /$ día y que aún no cuenta con clave en el Cuadro Básico y Catálogo de Medicamentos.

Modelo de evaluación

En el modelo se distinguen los siguientes cuatro estadios: tratamiento de SVM en pacientes con CaMa (sometidas a quimioterapia o terapia hormonal citostática con tamoxifeno), tratamiento de SVM en pacientes con progresión de $\mathrm{CaMa}$ (sometidas a cuidados paliativos para mejorar su calidad de vida), tratamiento de SVM en pacientes con antecedentes de CaMa (cáncer en remisión) y un estado absorbente que corresponde a la muerte de la paciente (Fig. 1). Las transiciones entre estos estados se dan en ciclos que tuvieron una duración de 3 meses, dado que este es el periodo mínimo para el cual todos los estudios clínicos a partir de los cuales se extrajeron datos de eficacia de las alternativas 
Tabla 1 Probabilidades de transición y AVAC's correspondientes a cada alternativa farmacológica considerada en el modelo
AVAC's: Años de vida ajustados por calidad, CaMa: Cáncer de mama, MPA/EC:

medroxiprogesterona + estrógenos conjugados

\begin{tabular}{lll}
\hline Variable & Valor & Fuente \\
\hline Progresión del estadio pacientes con tratamiento de CaMa & 0,4959 & {$[26]$} \\
Remisión a tratamiento de CaMa (probabilidad de recidivar) & 0,1100 & {$[27]$} \\
Tratamiento a tratamiento paliativo & 0,2865 & {$[26]$} \\
Remisión a tratamiento paliativo & 0,0001 & {$[26]$} \\
Muerte por CaMa en pacientes con tratamiento del CaMa & 0,1354 & {$[26]$} \\
Muerte de CaMa en "remisión de la enfermedad" & 0,0001 & {$[28]$} \\
Muerte por CaMa en "tratamiento paliativo" & 0,5200 & {$[29]$} \\
Permanecer en el estado de remisión & 0,8800 & {$[27]$} \\
Permanecer en el tratamiento de CaMa & 0,7895 & {$[26]$} \\
Permanecer en tratamiento paliativo & 0,1399 & {$[26]$} \\
Alternativa de tratamiento & AVACs ganados & \\
Desvenlafaxina & Promedio: & \\
Tibolona & 0,150 & \\
Estrógenos conjugados & 0,156 & \\
MPA/EC & 0,153 & \\
\hline
\end{tabular}

reportan resultados [12-16]. Las probabilidades de transición entre los estados del modelo se encuentran en función del abandono del esquema farmacológico asignado para el manejo de los SVM y de la historia natural del CaMa. El análisis finaliza cuando la cohorte de pacientes que ingresa al mismo completa 20 ciclos (equivalente a 5 años). El modelo también considera la posibilidad de que se presenten eventos adversos asociados al esquema farmacológico para manejo de SVM.

Probabilidades de transición

La estimación de la probabilidad de abandono en el tiempo del tratamiento con TIB, MPA/EC, EC y DVS se basó en los resultados de estudios longitudinales [3, 17-25], los cuales fueron usados en un modelo exponencial de tasa de abandono desarrollado previamente [10]. El citado modelo refleja un dramático retiro del tratamiento farmacológico para SVM a partir de los primeros 3 meses de prescripción, hasta llegar a una tasa de retiro más estable a partir del segundo año.

Las probabilidades de transición entre los estados de salud considerados en el modelo se estimaron a partir de la revisión de la literatura [26-29] y se muestran en la Tabla 1. Se asumió que las probabilidades de transición son constantes en el tiempo.

\section{Incidencia de eventos adversos}

La frecuencia de presentación y tipo de evento adverso asociados a cada esquema de tratamiento de SVM se estimó a partir del análisis retrospectivo de expedientes clínicos $(n=140)$ pacientes en tratamiento por CaMa o antecedentes de CaMa atendidos en el IMSS entre marzo de 2009 a enero de 2010. La consistencia de la información recolectada se evaluó por medio de la revisión por parte de dos médicos oncólogos.

\section{Costes}

El presente estudio se realizó considerando la perspectiva del proveedor de servicios de salud, por lo que se toman en cuenta los costes directos en los que incurre el IMSS en el manejo de SVM en pacientes con CaMa o antecedentes de CaMa, tales como el tratamiento farmacológico para SVM, las pruebas de tamiz para elegibilidad de TRH, el coste asociado al tratamiento de los eventos adversos de cada esquema de atención (constituido por costes de hospitalización, consultas médicas, cirugías, estudios de laboratorio y gabinete, transfusiones sanguíneas y tratamiento farmacológico). Los costes unitarios de los recursos médicos fueron obtenidos de informes contables del IMSS [30]. Los costes de los fármacos para el manejo de SVM fueron extraídos del Catálogo local de medicamentos 2009-Hospital de Oncología, IMSS [31], a excepción del de DVS, que fue proporcionado por Pfizer S.A. de C.V. (Tabla 2). En el presente estudio, todos los costes se encuentran expresados en US\$ de 2011 (tipo de cambio 1,41 US\$/€).

Fuentes de efectividad y utilidad

Se realizó una búsqueda sistemática de literatura publicada entre 1990 y 2009 para documentos publicados en Inglés y Español dentro de las siguientes bases de datos elec- 
Tabla 2 Coste de manejo de eventos adversos y terapias farmacológicas para manejo de SVM (trimestral)

SVM: síntomas vasomotores, TRH: Terapia de reemplazo hormonal, DVS: desvenlafaxina, TIB: tibolona, EC: estrógenos conjugados, MPA/EC: medroxiprogesterona + estrógenos conjugados

\begin{tabular}{llll}
\hline Esquema de tratamiento & $\begin{array}{l}\text { Tratamiento } \\
\text { farmacológico de } \\
\text { SVM (US\$) }\end{array}$ & $\begin{array}{l}\text { Pruebas de tamiz } \\
\text { para elegibilidad de } \\
\text { TRH (US\$) }\end{array}$ & $\begin{array}{l}\text { Tratamiento de } \\
\text { eventos adversos } \\
\text { (US\$) }\end{array}$ \\
\hline DVS & 194,04 & - & 1,66 \\
TIB & 73,94 & 1,49 & 167,07 \\
EC & 28,72 & 1,49 & 176,04 \\
MPA/EC & 65,71 & 1,49 & 220,64 \\
Ningún tratamiento & - & - & - \\
\hline
\end{tabular}

trónicas: Artemisa, AdisInsight, Cochrane, Hermes, OvidMedline, Elsevier, Science Direct, Ebsco-Host data base, Ebsco E Journal Services y Springer Link. Los términos clave empleados en la búsqueda (y sus equivalentes en inglés) fueron: bochornos, síntomas vasomotores, terapia de reemplazo hormonal, CaMa, eventos adversos, coste-efectividad. Los anteriores términos se cruzaron con los siguientes: desvenlafaxina, estrógenos conjugados, medroxiprogesterona y tibolona. Se seleccionaron documentos que reportaran uno o varios de los siguientes resultados: Años de Vida Ajustados por Calidad (AVAC's), la reducción promedio en el número de bochornos, la frecuencia de presentación de eventos adversos, la tasa de abandono de tratamiento a SVM, así como las probabilidades de remisión, presentación de recidivas, supervivencia y muerte de pacientes con CaMa.

A partir de la revisión retrospectiva de expedientes, se estimó el impacto en la calidad de vida asociado a cada esquema de tratamiento de SVM mediante el uso de la escala de Cervantes [32]. Dado que el tratamiento de SVM no afecta la duración del cuadro de SVM, sino que modifica la calidad de vida de las pacientes, se usó la metodología descrita por Sassi [33] para estimar la ganancia en términos de AVAC's, considerando como referencia al ponderador de calidad de vida cuando no reciben tratamiento para SVM y el estado en que no se presentan los episodios de bochornos. Las estimaciones para cada tratamiento se presentan en la Tabla 1.

Medidas de efectividad y costes estimados

La efectividad del tratamiento fue valorada en términos del promedio de bochornos evitados por día por paciente después de 12 semanas de tratamiento y los AVAC's ganados por paciente. Los costes estimados por el modelo corresponden al monto promedio de la inversión institucional requerida para el manejo de SVM por paciente con diagnóstico o antecedentes de CaMa. Los costes y la efectividad estimados por el modelo se encuentran descontados considerando una tasa del $5 \%$ anual, de acuerdo a las indicaciones de la Guía para la conducción de estudios de evaluación económica para la actualización del Cuadro Básico de Insumos del Sector Salud en México [34].
Los resultados del análisis se expresan en términos de la razón de coste-efectividad incremental (RCEI), correspondiente a cada medida de efectividad y alternativa de tratamiento de SVM. Las RCEI's se calcularon como la diferencia entre el coste promedio estimado por paciente correspondiente a cada alternativa y el coste promedio estimado por paciente tratado con DVS, dividido por el número promedio de bochornos evitados o AVAC's ganados correspondiente a cada alternativa respecto al correspondiente estimado para DVS. Las RCEI's se encuentran expresadas en US\$/bochorno adicional evitado por día y en US\$/AVAC ganado.

Análisis de sensibilidad

Se realizó un análisis de sensibilidad del tipo probabilístico con los datos obtenidos del modelo Markov, con una cantidad de 10.000 iteraciones para garantizar robustez. A partir de este análisis se construyeron las curvas de aceptabilidad para simular la variación de la relación coste-efectividad al comparar DVS con los otros esquemas de tratamiento.

\section{Resultados}

El esquema farmacológico con el menor coste médico directo promedio por trimestre fue DVS (US\$195,70), el segundo menor coste le corresponde al manejo de SVM basado en EC (US\$206,25), $p<0,05$. Los esquemas farmacológicos basados en TIB y MPA/EC presentaron costes promedio mayores al manejo con DVS en US\$46,80 y US\$92,14, respectivamente.

La mayor efectividad, expresada como la mayor reducción en el número de bochornos por día la presentó TIB (7,66 eventos después de un periodo de 12 semanas de tratamiento). Sin embargo, al considerar el efecto que la tasa de abandono del tratamiento para el manejo de SVM en el horizonte de análisis completo (5 años), el esquema farmacológico basado en DVS presentó una mayor efectividad acumulada (dado que se constituye como la estrategia con la menor tasa de abandono de tratamiento para SVM). 
Tabla 3 Resultados de coste-efectividad. Grupo de pacientes sin útero
RCEI: Razón de coste-efectividad incremental, DVS: desvenlafaxina, TIB: tibolona, EC: estrógenos conjugados, AVAC: Año de vida ajustado por calidad

Tabla 4 Resultados de coste-efectividad. Grupo de pacientes con útero
RCEI: Razón de

coste-efectividad incremental, DVS: desvenlafaxina, TIB:

tibolona, MPA/EC:

medroxiprogesterona + estrógenos conjugados, AVAC: Año de vida ajustado por calidad

\begin{tabular}{|c|c|c|c|c|c|c|}
\hline $\begin{array}{l}\text { Alternativa de } \\
\text { tratamiento }\end{array}$ & $\begin{array}{l}\text { Coste promedio } \\
\text { (US\$) }\end{array}$ & $\begin{array}{l}\text { Efectividad (gan } \\
\text { en reducción de } \\
\text { bochornos) }\end{array}$ & & $\begin{array}{l}\text { Coste } \\
\text { incremental } \\
\text { (US\$) }\end{array}$ & $\begin{array}{l}\text { Efectividad } \\
\text { incremental }\end{array}$ & $\begin{array}{l}\text { RCEI } \\
\text { (US\$/bochorno } \\
\text { adicional } \\
\text { evitado) }\end{array}$ \\
\hline DVS & $3.011,46$ & $3.109,56$ & & - & - & - \\
\hline $\mathrm{EC}$ & $3.403,56$ & $2.668,11$ & & 392,10 & $-441,45$ & Dominado \\
\hline TIB & $3.636,07$ & $2.297,82$ & & 624,61 & $-811,74$ & Dominado \\
\hline $\begin{array}{l}\text { Alternativa de } \\
\text { tratamiento }\end{array}$ & $\begin{array}{l}\text { Coste promedio } \\
\text { (US\$) }\end{array}$ & $\begin{array}{l}\text { Utilidad } \\
\text { (AVAC's) }\end{array}$ & \multicolumn{2}{|c|}{$\begin{array}{l}\text { Coste incremental } \\
\text { (US\$) }\end{array}$} & $\begin{array}{l}\text { Utilidad } \\
\text { incremental } \\
\text { (AVAC's) }\end{array}$ & $\begin{array}{l}\text { RCEI } \\
\text { (US\$/AVAC } \\
\text { ganado) }\end{array}$ \\
\hline DVS & $3.011,46$ & 0,80 & \multicolumn{2}{|l|}{ - } & - & - \\
\hline $\mathrm{EC}$ & $3.403,56$ & 0,64 & \multicolumn{2}{|c|}{392,10} & $-0,16$ & Dominado \\
\hline TIB & $3.636,07$ & 0,59 & \multicolumn{2}{|c|}{624,61} & $-0,21$ & Dominado \\
\hline
\end{tabular}

\begin{tabular}{|c|c|c|c|c|c|}
\hline $\begin{array}{l}\text { Alternativa de } \\
\text { tratamiento }\end{array}$ & $\begin{array}{l}\text { Coste promedio } \\
\text { (US\$) }\end{array}$ & $\begin{array}{l}\text { Efectividad (ganancia } \\
\text { en reducción de } \\
\text { bochornos) }\end{array}$ & $\begin{array}{l}\text { Coste } \\
\text { incremental } \\
\text { (US\$) }\end{array}$ & $\begin{array}{l}\text { Efectividad } \\
\text { incremental }\end{array}$ & $\begin{array}{l}\text { RCEI } \\
\text { (US\$/bochorno } \\
\text { adicional } \\
\text { evitado) }\end{array}$ \\
\hline DVS & $3.169,29$ & $3.109,56$ & - & - & - \\
\hline TIB & $3.894,38$ & $2.297,82$ & 725,09 & $-811,74$ & Dominado \\
\hline MPA/EC & $4.114,98$ & $1.344,69$ & 945,69 & $-1.764,87$ & Dominado \\
\hline $\begin{array}{l}\text { Alternativa de } \\
\text { tratamiento }\end{array}$ & $\begin{array}{l}\text { Coste promec } \\
\text { (US\$) }\end{array}$ & $\begin{array}{l}\text { Utilidad } \\
\text { (AVAC's) }\end{array}$ & $\begin{array}{l}\text { Coste } \\
\text { incremental } \\
\text { (US\$) }\end{array}$ & $\begin{array}{l}\text { Utilidad } \\
\text { incremental } \\
\text { (AVAC's) }\end{array}$ & $\begin{array}{l}\text { RCEI } \\
\text { (US\$/AVAC } \\
\text { ganado) }\end{array}$ \\
\hline DVS & $3.011,46$ & 0,80 & - & - & - \\
\hline TIB & $3.636,07$ & 0,59 & 624,61 & $-0,21$ & Dominado \\
\hline MPA/EC & $3.843,20$ & 0,42 & 831,74 & $-0,38$ & Dominado \\
\hline
\end{tabular}

Los resultados del análisis en el grupo de pacientes sin útero (comparación de TIB y EC respecto de DVS) se muestran en la Tabla 3, los resultados del análisis en el grupo de pacientes con útero (TIB y MPA/EC respecto de DVS) se muestran en la Tabla 4. Los resultados del análisis en el grupo de pacientes con TRH contraindicada se muestran en la Tabla 5. En la Tabla 3 se observa que el coste promedio del tratamiento por paciente a lo largo de 5 años con DVS fue US\$3.011,46, que fue menor entre US\$392,09 y US $\$ 624,61$ respecto del coste asociado a las otras alternativas. El volumen de reducción de bochornos en el grupo de pacientes sin útero tratado con DVS a lo largo del horizonte fue de $3.109,56$ eventos, equivalente a $16,5 \%$ y $35,3 \%$ más que las estimadas para EC y TIB, respectivamente.

Un resultado similar se observa con los valores de utilidad promedio que una paciente acumula a lo largo del horizonte de análisis: la ganancia en AVAC's acumulada con DVS fue de 0,8 (correspondiente a la mayor estimación). Relacionando el perfil de costes de cada alternativa con sus correspondientes salidas en salud, se tiene que DVS es una alternativa no-hormonal coste-ahorradora o dominante respecto de las demás alternativas en este grupo de pacientes.

Los resultados del grupo de pacientes con útero y del grupo de pacientes que tienen contraindicado el uso de TRH presentaron la misma tendencia de los resultados presentados en la Tabla 3. En estos grupos de pacientes, el manejo de SVM con DVS es la estrategia a la cual se asocian los mejores perfiles de coste y de efectividad, ya sea que esta se mida en términos de la reducción en el número de bochornos por día o del número de AVAC's ganado por paciente a lo largo de 5 años. Específicamente, el manejo de SVM constituye una estrategia coste-ahorradora cuando se compara con las consecuencias del uso de TIB, MPA/EC y EC y coste-efectiva respecto de la estrategia de no usar ningún tratamiento farmacológico para el manejo de SVM (mujeres con contraindicación de TRH).

Los resultados del análisis cuando se consideró la variabilidad en la respuesta clínica de cada uno de los tratamien- 
Tabla 5 Resultados de coste-efectividad. Grupo de pacientes en los que la TRH está contraindicada

RCEI: Razón de coste-efectividad incremental, DVS: desvenlafaxina, TIB: tibolona, AVAC: Año de vida ajustado por calidad

\begin{tabular}{|c|c|c|c|c|c|}
\hline $\begin{array}{l}\text { Alternativa de } \\
\text { tratamiento }\end{array}$ & $\begin{array}{l}\text { Coste promedio } \\
\text { (US\$) }\end{array}$ & $\begin{array}{l}\text { Efectividad (ganancia } \\
\text { en reducción de } \\
\text { bochornos) }\end{array}$ & $\begin{array}{l}\text { Coste } \\
\text { incremental } \\
\text { (US\$) }\end{array}$ & $\begin{array}{l}\text { Efectividad } \\
\text { incremental }\end{array}$ & $\begin{array}{l}\text { RCEI } \\
\text { (US\$/bochorno } \\
\text { adicional } \\
\text { evitado) }\end{array}$ \\
\hline DVS & 3.011 & $3.109,56$ & - & - & - \\
\hline Sin tratamiento & 1.825 & 355,76 & -1.186 & $-2753,8$ & 0,4307 \\
\hline $\begin{array}{l}\text { Alternativa de } \\
\text { tratamiento }\end{array}$ & $\begin{array}{l}\text { Coste promed } \\
\text { (US\$) }\end{array}$ & $\begin{array}{l}\text { Utilidad } \\
\text { (AVAC's) }\end{array}$ & $\begin{array}{l}\text { Coste } \\
\text { incremental } \\
\text { (US\$) }\end{array}$ & $\begin{array}{l}\text { Utilidad } \\
\text { incremental } \\
\text { (AVAC's) }\end{array}$ & $\begin{array}{l}\text { RCEI } \\
\text { (US\$/AVAC } \\
\text { ganado) }\end{array}$ \\
\hline DVS & 3.011 & 0,8 & - & - & - \\
\hline Sin tratamiento & 1.825 & 0,05 & -1.186 & $-0,75$ & 1.581 \\
\hline
\end{tabular}

A

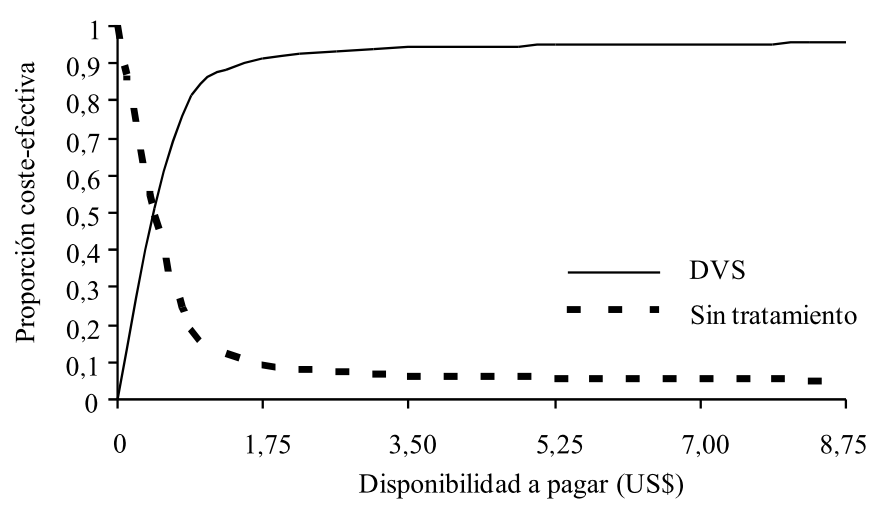

Figura 2 Curvas de aceptabilidad del manejo de síntomas vasomotores (SVM) en pacientes con terapia de reemplazo hormonal (TRH) contraindicada. A Efectividad expresada como eventos de bo-

tos asociados sirven de base para la generación de las curvas de aceptabilidad. Estas curvas brindan información acerca de la probabilidad de que una estrategia dada sea costeefectiva a una disponibilidad a pagar dada (la cantidad de recursos económicos que el pagador estaría dispuesto a invertir para obtener una unidad adicional de efectividad). Con fines de claridad solo se muestran las curvas de aceptabilidad correspondientes a la población de pacientes con restricción de uso de TRH (en ambas medidas de efectividad: reducción del número de bochornos por día y AVAC's ganados, Fig. 2, panel A y B, respectivamente). En ambos casos se observa que la mayor probabilidad de que DVS sea coste-efectiva se alcanza a disponibilidades a pagar (DAP) relativamente bajas: a partir de una DAP de US $\$ 1,75$ por bochorno adicional evitado, la probabilidad de que DVS sea una estrategia coste-efectiva es de por lo menos el $90 \%$ (Fig. 2, panel A). En el panel B de la Fig. 2 se observa que a partir de una DAP de US $\$ 1.750$ por AVAC ganado, DVS es la alternativa con una probabilidad de al menos $50 \%$ de ser costeefectiva.
B

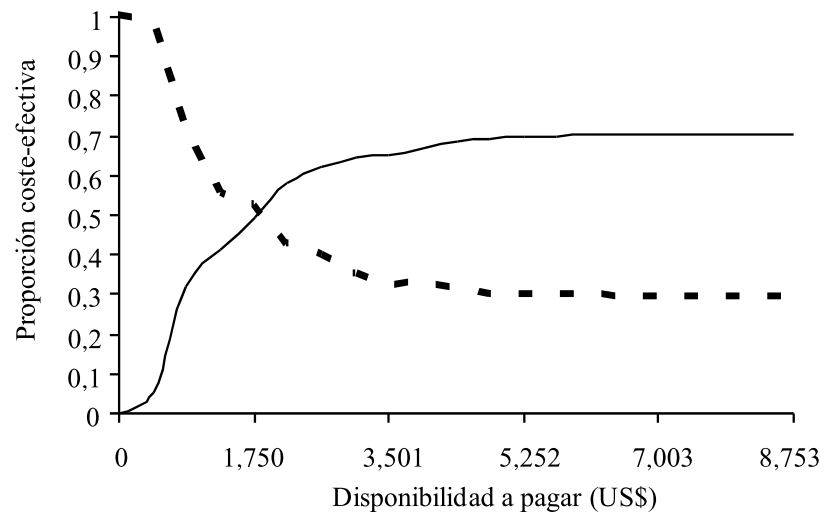

chornos adicionales evitados. B Efectividad expresada como Años de vida ajustados por calidad (AVAC's) ganados. DVS: desvenlafaxina

\section{Discusión}

En el presente estudio se estimaron los beneficios potenciales asociados al uso de diversas alternativas farmacológicas para el manejo de SVM en el IMSS. Los beneficios fueron estimados tanto en la mejora de calidad de vida de mujeres con CaMa o antecedentes con CaMa, como en las consecuencias económicas correspondientes al uso de cada alternativa farmacológica. Los resultados del estudio muestran que, en el manejo de SVM en tres grupos de pacientes (histerectomizadas, no histerectomizadas y aquellas en las que el uso de TRH se encuentra contraindicado), DVS constituye una alternativa no-hormonal que generaría ahorros institucionales.

Estos ahorros son consecuencia principalmente de dos aspectos: en primer lugar, un menor coste en los pacientes que utilizan DVS, dado por la menor utilización de recursos médicos empleados en el manejo de eventos adversos asociados a los otros esquemas farmacológicos empleados en el manejo de SVM, principalmente sangrado vaginal. En segundo lugar, el modelo estimó que las pacientes que utilizan 
DVS tienen la menor tasa de abandono de tratamiento a manejo de SVM, lo que al final del horizonte temporal se ve reflejado en un mayor nivel de efectividad o utilidad.

En este estudio se determinó que el coste por el manejo de eventos adversos asociados a la TRH por paciente al trimestre es de US $\$ 167,07$ para TIB, US $\$ 176,04$ para EC y de US\$220,64 para MPA/EC. En el presente estudio se estimó que el coste de manejo de los eventos adversos producidos por DVS es menor al $1 \%$ del coste total del esquema farmacológico basado en esta alternativa. El coste del manejo de eventos adversos en las alternativas de TRH analizadas en el presente estudio resultó ser la parte mayoritaria del coste total del manejo de SVM con estas alternativas, constituyendo el $70 \%$ o más del coste total del tratamiento anual. Esta proporción es mayor a la estimada por Plumb y Guest [35], quienes determinaron que el $47 \%$ del coste total de la TRH estuvo constituido por el manejo de eventos adversos, aunque solo considerando la contribución de tres eventos indeseables.

Dada la preocupación que representa administrar TRH en pacientes con CaMa o con antecedentes de CaMa para el manejo de SVM, el análisis de la población que tiene contraindicado el uso de TRH realizado en el presente estudio, cobra una importancia particular. En este grupo de pacientes, la RCEI del manejo farmacológico de SVM con DVS respecto de no brindar tratamiento alguno, fue de US $\$ 1.581$ por AVAC ganado. Este valor es comparable a los resultados encontrados por Zethraeus y cols. [36] en Suecia: (RCEI entre US $\$ 800$ a US $\$ 1.280$ por AVAC ganado).

Una de las principales limitantes del estudio fue que a pesar de los esfuerzos solo se consiguió un número limitado de pacientes cuyo manejo de SVM estuvo dado con EC y MPA/EC. Esto se debe a que, aunque queda al criterio del médico la utilización de la TRH en pacientes sin marcadores hormonales, en la práctica el uso de TRH es limitado.

Un importante supuesto sobre el que descansan los resultados del presente estudio es que la tasa de abandono de cada esquema de atención de SVM se ajustó a un modelo exponencial, para subsanar la limitación de datos longitudinales; futuros estudios que realicen seguimiento de pacientes a largo plazo permitirán tener los datos necesarios para relajar esta suposición.

En conclusión, el uso de desvenlafaxina a una dosis de $100 \mathrm{mg}$ /día para el manejo de SVM en mujeres con CaMa o antecedentes de CaMa en comparación con la TRH es una alternativa no-hormonal coste-ahorradora al reducir el uso de recursos médicos destinado al manejo de eventos adversos y reducir también la tasa de abandono del tratamiento de SVM. Respecto de la no administración de terapia alguna, desvenlafaxina constituye una alternativa coste-efectiva para el manejo de SVM.

Financiación y conflicto de intereses Esta investigación se realizó con el apoyo financiero de Pfizer S.A. de C.V. (México), sin que esto haya generado algún tipo de compromiso legal y/o sobre los resultados de la misma. Al momento de la realización del estudio Joaquín Federico Mould-Quevedo era empleado de Pfizer S.A. de C.V., Miguel Angel Ramírez era empleado de CPP Customized Premium Products S.A. de C.V. Miguel Angel Ramírez, Gustavo Peniche y Carlos Nuño han actuado como consultores para Roche, Teva, Schering Plough, Pfizer, BMS, GSK y Lilly.

Open Access This article is distributed under the terms of the Creative Commons Attribution License which permits any use, distribution, and reproduction in any medium, provided the original author(s) and the source are credited.

\section{Bibliografía}

1. North American Menopause Society (NAMS). Treatment of menopause-associated vasomotor symptoms: position statement of the North America Menopause Society. Menopause. 2004;11(1):11-33.

2. Gold EB, Sternfeld B, Kelsey JL, et al. Relation of demographic and lifestyle factors to symptoms in a multi-racial/ethnic population of women 40-55 years of age. Am J Epidemiol. 2000;152(5):463-73.

3. Velasco-Murillo V, Fernández-GárateI H, Ojeda-Mijares RI, et al. Conocimientos, experiencias y conductas durante el climaterio y la menopausia en las usuarias de los servicios de medicina familiar del IMSS. Rev Med Inst Mex Seguro Soc. 2007;45(6):549-56.

4. Xydakis MA, Sacas EG, Mastorakos G. Hormone replacement therapy in breast cancer survivors. Ann NY Acad Sci. 2006;1092:349-60.

5. Mortimer JE, Flatt SW, Parker BA, et al. Tamoxifen, hot flashes and recurrence in breast cancer. Breast Cancer Res Treat. 2008;108(3):421-6.

6. Instituto Mexicano del Seguro Social. Dirección de Prestaciones Médicas. Unidad de Atención Médica. Coordinación de Unidades Médicas de Alta Especialidad. División de Excelencia Clínica. Guía de práctica clínica. Atención del climaterio y menopausia. México: Instituto Mexicano del Seguro Social. 2009.

7. Lilue M, Palacios S. Tratamiento no-hormonal de los síntomas vasomotores durante la menopausia: papel de la desvenlafaxina. Ginecol Obstet Méx. 2009;77(10):475-81.

8. Botteman MF, Shah N, Lian J, et al. A cost-effectiveness evaluation of two continuous combined hormone therapies for the management of moderate to severe vasomotor symptoms. Menopause. 2004;11(3):343-55.

9. Coyle D, Cranney A, Tugwell P. Economic evaluation of norethisterone acetate/ethinylestradiol $\left(\mathrm{FemHRT}^{\circledR}\right)$ for women with menopausal symptoms. Pharmacoeconomics. 2003;21(9):661-9.

10. Diaby V, Perreault S, Lachaine J. Economic impact of Tibolone compared with continuous combined hormone replacement therapy in the management of climateric symptoms in postmenopausal women. Maturitas. 2007;58(1):138-49.

11. Consejo de Salubridad General. Cuadro Básico y Catálogo de Medicamentos Edición 2009. Diario Oficial de la Federación (segunda y tercera secciones). México 23 abril del 2010.

12. Archer DF, Dupont CM, Constantine GD, et al. Desvenlafaxine for the treatment of vasomotor symptoms associated with menopause: a double-blind, randomized, placebo-controlled trial of efficacy and safety. Am J Obstet Gynecol. 2009;200(3):238.e1.

13. Biglia N, Torta R, Roagna R, et al. Evaluation of low-dose venlafaxine hydrochloride for the therapy of hot flushes in breast cancer survivors. Maturitas. 2005;52(1):78-85.

14. Landgren MB, Helmond FA, Engelen S. Tibolone relieves climacteric symptoms in highly symptomatic women with at least seven hot flushes and sweats per day. Maturitas. 2005;50(3):222-30. 
15. Utian WH, Lederman SA, Williams BM, et al. Relief of hot flushes with new plant-derived 10-component synthetic conjugated estrogens. Obstet Gynecol. 2004;103(2):245-53.

16. Odmark IS, Bäckström T, Jonsson B, et al. Well-being at onset of hormone replacement therapy: comparison between two continuous combined regimens. Climacteric. 2004;7(1):92-102.

17. Pilon D, Castilloux AM, Lelorier J. Estrogen replacement therapy: determinants of persistence with treatment. Obstet Gynecol. 2001;97(1):97-100.

18. Ravnikar VA. Compliance with hormone therapy. Am J Obstet Gynecol. 1987;156:1332-4.

19. Sullivan JM. Estrogen replacement therapy. Am J Med. 1996;101(Suppl A):56S-60S.

20. Simon J, Wysocki S, Brandman J, et al. A comparison of therapy continuation rates of different hormone replacement agents a 9 months retrospective, longitudinal analysis of pharmacy claims among new users. Menopause. 2003;10(1):37-44.

21. Hammar M, Christau S, Nathorst-Boos J, et al. A double blind, randomized trial comparing the effects of tibolone and continuous combined hormone replacement therapy in postmenopausal women with menopausal symptoms. Br J Obstet Gynaecol. 1998;105(1):904-11.

22. Perrry R, Desvenlafaxine CM. A new serotonin norepinephrine reuptake inhibitor for the treatment of adults with major depressive disorder. Clin Ther. 2009;31(1):1375-404.

23. Carpenter J, Storniolo AM, Johns S, et al. Randomized, double blind, placebo controlled crossover trials of venlafaxine for hot flashes after breast cancer. The Oncologist. 2007;12(1):124-35.

24. Kumari M, Stafford M, Marmot M. The menopausal transition was associated in a prospective study with decreased health functioning in women who report menopausal symptoms. J Clin Epidemiol. 2005;58(7):719-27.

25. Speroff L, Gass M, Constantine G, et al. Efficacy and tolerability of desvenlafaxine succinate treatment for menopausal vasomotor symptoms: a randomized controlled trial. Obstet Gynecol. 2008;111(1):77-87.

26. Knaul FM, Arreola-Ornelas H, Velazquez E, et al. Costo de la atención médica del cáncer mamario: el caso del Instituto Mexicano del Seguro Social. Salud pública Mex. 2009;51(sup 1):s287s295.
27. Aguilar E, de la Flor M, Ballesteros M, et al. Evaluación de los factores pronósticos de cáncer de mama. Ginecol Clín Quir. 2001;2(4):2000.

28. Knaul FM, Lozano R, Arreola H, et al. México: Numeralia del cáncer de mama. Fundación Mexicana para la Salud 2008. Disponible en: http://www.funsalud.org.mx/competitividad/ NUMERALIA\%20CAMA.pdf [Consultado el 10 enero 2010].

29. Oruezabal M. Factores pronósticos y predictivos de la supervivencia global y libre de progresión de pacientes con cáncer de mama metastásico. Universidad Complutense de Madrid, Madrid, España. 2002.

30. Instituto Mexicano del Seguro Social. ACUERDO ACDO. SA2.HCT.250209/41.P.DF, por el que se aprueban los Costos Unitarios por Nivel de Atención Médica para el año 2009 y la Base de Cálculo para la Actualización de los Costos Unitarios por Nivel de Atención Médica para el año 2009. Diario Oficial de la Federación (segunda sección), 26 de marzo de 2009.

31. Instituto Mexicano del Seguro Social. Hospital de Especialidades. Catálogo de Medicamentos 2009.

32. Palacios S, Ferrer-Barrientos J, Parrilla JJ, et al. Calidad de vida relacionada con la salud en la mujer española durante la premenopausia y posmenopausia. Desarro Validación Escala Cervantes. Med Clin Barc. 2004;122(6):205-11.

33. Sassi F. Calculating QALYs, comparing QALY and DALY calculations. London: The London School of Economics and Political Science. 2006.

34. Comisión Interinstitucional del Cuadro Básico de Insumos del Sector Salud. Guía para la conducción de estudios de evaluación económica para la actualización del Cuadro Básico de Insumos del Sector Salud en México. Consejo de Salubridad General, México. 2008.

35. Plumb JM, Guest J. Economic impact of tibolone compared with continuos combined hormone replacement therapy. Pharmacoeconomics. 2000;18(5):477-86.

36. Zethraeus N, Borgström F, Jönsson B, et al. Reassessment of the cost-effectiveness of hormone replacement therapy in Sweden: results based on the Women's Health Initiative randomized controlled trial. J Int J Technol Assess Health Care. 2005;21(4):433-41. 